\title{
Automated atom-by-atom 3D reconstruction of field ion microscopy data
}

\author{
Michal Dagan*, Baptiste Gault*,**, George D W Smith*, Paul A J Bagot*, Michael P Moody*
}

Corresponding author: michael.moody@materials.ox.ac.uk

* Department of Materials, University of Oxford, Parks Road, Oxford, OX1 3PH UK

** Max Planck Institut für Eisenforschung GmbH, Max-Planck Straße 1, 40237 Düsseldorf, Germany

\section{Abstract}

An automated procedure has been developed for the 3D reconstruction of field ion microscopy (FIM) data that maintains the atomistic nature of the technique. FIM characterises individual atoms on the surface of a specimen, that is evolving subject to the process of field evaporation, in a series of 2D time-ordered images. It's unique spatial resolution enables the direct imaging of crystal defects, as small as single vacancies. In order to exploit the full potential of FIM, automated analysis tools are required. The reconstruction algorithm developed in this work relies on minimal assumptions and is sensitive to the atomic coordinates of all imaged atoms. It tracks the atoms across a sequence of FIM images and allocates each one to its respective crystallographic plane. The result is a highly accurate 3D lattice-resolved reconstruction. The procedure is applied here to over 2000 tungsten atoms, including several ionimplanted planes of the sample. The approach is also adapted for the analysis of carbides in a bearing steel matrix, to demonstrate its potential applicability to the study of a broader range of materials. A vast amount of additional information is collected during the experiment. This information can underpin advanced analyses such as automated detection of 'out of sequence' events, sub-angstrom displacements of atoms and defects effects on neighbouring atoms. These analyses have the potential to reveal new insights into the field evaporation process and contribute to improving accuracy and scope of 3DFIM and atom probe characterisation.

Keywords: field ion microscopy, 3D reconstruction, atomic resolution, crystal defects 


\section{Introduction}

Materials performance is intimately linked to microstructural architecture at the atomic-scale. The continued development of models to explain mechanical properties, and ultimately design new and improved materials is therefore critical for an increasing number of applications. However, directly imaging in 3D, not only all constituent atoms within a material, but also vacancies and other types of defects, has proven to be beyond the resolution limit of most current conventional microscopy techniques, and remains a formidable challenge. For example, such atomic-scale characterization capabilities are essential in radiation damage studies. A remaining hurdle in the development of nuclear fusion power generation is the lack of materials that can withstand the extreme operating conditions inside the reactor [1], [2]. A thorough understanding of radiation-induced degradation mechanisms of the internal microstructure under such conditions is therefore of paramount importance.

Recent efforts in electron microscopy have proposed complex solutions to atomic-scale tomographic imaging of materials [3]-[5]. Field ion microscopy (FIM) was the first microscopy technique to image individual atoms[6], lattice defects in metals including single vacancies[7], interstitials and extended dislocations [8], [9]. In contrast to other high-resolution microscopy techniques, FIM relies on the ionization of inert gas atoms from prominent positions on the specimen surface exploiting an intense electric field. The electric field is generated at the apex of the very sharp needle-shaped specimen[10] maintained at cryogenic temperature and biased to a few $\mathrm{kV}$. The divergent electric field drives the ions away from the specimen and onto a phosphor screen, creating a highly magnified, quasi-stereographic, two-dimensional projection of the atomic arrangement at the surface of the specimen. A schematic illustration of the FIM setup is shown in Figure 1a. Voltage pulses superimposed on the DC voltage enable a highly-controlled removal of atoms constituting the specimen itself via field evaporation. Although each FIM image is intrinsically 2D, this procedure results in a series of images, each representing a 'snapshot' of the surface over the course of the field evaporation process. As the depth of the specimen is probed by the removal of the constituent atoms, the images progressively provide $3 \mathrm{D}$ atomic information on the specimen.

Ground-breaking research in the 1960's and 1970's demonstrated the unique insights FIM can bring into the study of fine-scale radiation damage. In a series of experiments, Seidman and co-workers directly imaged the early stages of radiation damage, manifesting as very small, atomic-scale defects in the crystal lattice by developing a novel 3D FIM approach [11]-[14]. In these experiments, the nature and spatial distribution of single vacancies and larger vacancy clusters were measured across 3D volumes of the specimens. Since these experiments pre-dated digital data collection, FIM images were recorded on film and analysed manually. The 3D reconstruction of the analysed volumes was therefore heavily restricted by the time-consuming manual analysis.

More recently, 3D FIM was further advanced by the development of procedure for the digital-stacking of time-ordered images [15], applied, for example, to the investigation of GP zones in steels [16], nitrides in Fe-Cr alloys [17] and fine scale precipitates in CuFeNi [18] . Theoretically these images evolve, atom-byatom and layer-by-layer, where each slice approximately represents a constant increment in the depth of the analysis. The stacking parameters can also be calibrated to match prominent crystallographic features in the specimen (i.e. spacing between atomic planes). Bending of the stack can be performed to account for the highly curved specimen shape. This procedure proved highly successful to uncover features that did not display clear contrast on the 2D FIM images but could be directly observed in a cross section of 
the 3D FIM reconstruction, such as dislocations extending along the tip axis [15]. However, this image stacking approach can become limiting for the precise identification of individual point defects in the matrix.

In recent years FIM has been somewhat overshadowed by the emergence of wide-field-of-view atom probe tomography (APT). APT is similarly based on the concept of field evaporation of specimen atoms from the surface [10].The evaporated ions strike a position-sensitive detector and their time-of-flight is measured, informing as to their chemical identity. Ultimately, using the digitally-recorded detector coordinates and a reverse-projection model, a 3D atomistic reconstruction of the tip is obtained [19].

The automated 3D atom-by-atom imaging combined with highly resolved elemental discrimination offered by APT provides significant advantages in comparison to FIM. However, these strengths are achieved at the expense of detection efficiency, i.e. due to the incorporation of the microchannel plates in current APT position sensitive detectors, a significant number of ions striking the detector are not registered by the experiment. While the maximum achievable detection efficiency in the latest generation of commercial atom probe instruments is reported to reach $80 \%$, in the case of many existing reflectronfitted instruments up to $65 \%$ of the atoms evaporated from the specimen are stochastically omitted from the final analysis [20], [21]. The incomplete nature of APT information makes it impossible to directly image every atomic site on the lattice and requires the application of advanced methods to characterise individual sites [22]. Reliable characterization of atomic-sized defects such as vacancies and interstitials is therefore not possible with APT.

In contrast, FIM is not subject to the same limitations of detection efficiency. The imaging of each surface atom in the analysis is effectively formed by the combined contributions of thousands of imaging gas ions per second. The data therefore contains full representation of all atoms in the analysed volume, and enables the detection of defects as small as vacancies that could not be characterized with APT [23].

In order to fully utilize the unique imaging capabilities of FIM and explore larger volumes accurately and consistently, this study introduces the first automated 'atom-by-atom' approach to 3D FIM data reconstruction. The procedure relies on minimal underlying assumptions, is sensitive to atomic information and sub-angstrom displacements in the imaged positions of the atoms, and results in an atomically resolved 3D reconstruction of the crystalline lattice. The proposed reconstruction procedure is demonstrated here for the analysis of tungsten, currently a leading candidate for plasma-facing components of nuclear fusion reactors [24]-[26]. However, the method is applicable to a wide range of materials which can be imaged by FIM such as steels and catalyst metals.

The approach developed here for reconstructing FIM data maintains the truly atomistic nature of the information in FIM images. We demonstrate the procedure for FIM analysis of the atomically resolved $[2,2,2]$ pole of a tungsten needle, characterised by 7771 images, describing the evaporation of 69 crystallographic layers in depth into the material and comprising 2450 tungsten atoms. We demonstrate how each atom can be automatically identified across the sequence of images, its position tracked across all images, from the time it is first revealed on the surface of specimen to the instant when it is fieldevaporated. Ultimately, all of this information is brought together to reconstruct original position of each atom on the crystal lattice and create a complete atomistic 3D image of the analysed volume. The main stages of the reconstruction algorithm are demonstrated, and significant challenges in the reconstruction procedure discussed. To explore its implementation to more complex material systems and datasets, a second reconstruction case study of carbides in a bearing steel is discussed. 


\section{Experiment}

A tungsten FIM sample was electropolished in a $5 \% \mathrm{NaOH}$ solution with the application of $5-8 \mathrm{~V}$ of $\mathrm{AC}$ voltage from a tungsten wire oriented along the [011] direction, in a one-step electropolishing procedure. The final specimen apex radius was estimated to be approximately $20 \mathrm{~nm}$. The sample was subject to selfion implantation to a dose consistent with primary stages of radiation damage, where damage cascades caused by the implantation of individual ions are not interacting. However, since the focus of this paper is to illustrate the $3 \mathrm{D}$ reconstruction algorithm, the majority of results presented in this study characterise an un-damaged region of this sample.

FIM experiments were performed on a 3DAP-LAR instrument, where a phosphor screen dedicated to FIM mode is installed, and images are taken digitally with a AVT stingray camera. Experiment was performed at a temperature of $50 \mathrm{~K}$, using voltage pulsing at a repetition rate of $100 \mathrm{kHz}$ for the controlled evaporation of the specimen surface. Ultra-high purity helium was used as the imaging gas, and was kept at a constant pressure of $3 \times 10^{-5} \mathrm{mbar}$.

For the second reconstruction demonstration, samples were electropolished from a M50 bearing steel in a two step process, with a $25 \%$ perchloric acid in acetic acid used for the first stage, and a $2 \%$ perchloric acid in butoxyethanol used for the second stage. Neon was used as the imaging gas for FIM images, at a pressure of $3 \times 10^{-5} \mathrm{mbar}$.

The DC voltage in both cases was determined according to the best imaging conditions, and was raised throughout the measurement as atoms were removed and the radius of the specimen increased. The voltage pulser was set to maintain controlled evaporation, with an average value of $25 \%$ of the applied DC voltage at each point in time. Images were taken at a rate of $15 \mathrm{fps}$, in SNR mode of 8 images, (in this mode the camera takes and averages over a set number of images to optimize the signal-to-noise ratio) and continuously recorded the arrangement of atoms on the evolving surface of the samples.

\section{FIM images}

In figure 1a, a typical FIM image of the tungsten tip is shown. Each bright spot on the image is representative of the position of an atom protruding from the surface of the sample. It is created by thousands of imaging gas atoms ionized at this position and projected by the field onto the phosphor screen. The crystallographic nature of the sample is clearly reflected in the image. A well-defined pole structure is apparent with a symmetry characteristic to the BCC tungsten lattice, which can be indexed appropriately.

The imaging and evaporation sequence of the atoms is fundamental to the reconstruction procedure. The terrace patterns are created as a result of the higher electric field generated above the more exposed surface sites at kink positions around the poles. Outer terrace atoms protrude to a greater extent than inner plane atoms, therefore higher gas ion current will be generated at these locations, giving brighter imaging and desired surface contrast. As the evaporation of constituent atoms progresses, the outer terrace is removed first due to the higher electric field, exposing the inner plane atoms for imaging. The layer-by-layer field evaporation process is demonstrated in Figure $1 \mathrm{~b}$, and in the supplementary videos.

As the imaging through the depth of the specimen continues, the repeating pattern provides a distinct metric as required for the automatic detection of the number of planes evaporated within a stack of FIM images. Figure $1 \mathrm{c}$ presents the integrated intensity measured from the centre pixels of the $[2,2,2]$ pole 
across the first 500 consecutive FIM images. Due to the imaging sequence outlined in figure $1 \mathrm{~b}$, each crystallographic layer will first exhibit low intensity in the middle of the pole, followed by the sequential imaging of atoms at the centre of the pole, corresponding to the sudden increase in intensity in figure 1c. Once the final atoms are evaporated, and a lower layer is exposed to the surface, the intensity at the centre of the pole drops again. This drop in intensity is usually sharp, since the final 3 atoms of a plane tend to field evaporate almost simultaneously, or at least within a time delay that the experiment cannot resolve.

Hence, each cycle of intensity intervals as shown in figure $1 \mathrm{c}$ represents the imaging and complete evaporation of a crystallographic layer. From this plot it can be concluded that in the first 500 FIM images, $6[2,2,2]$ planes are imaged, and 5 of them have been fully evaporated. From the intensity drop points on the plot, the final FIM image in the sequence that corresponds to a particular plane can be determined, i.e. one image prior to point labelled 4 in the example in figure $1 \mathrm{~b}$, c. Since the images are taken at a constant rate, the width of each intensity interval corresponds to the evaporation time of each plane. This will evolve through the experiment due to changing field conditions as the shape of the specimen evolves.
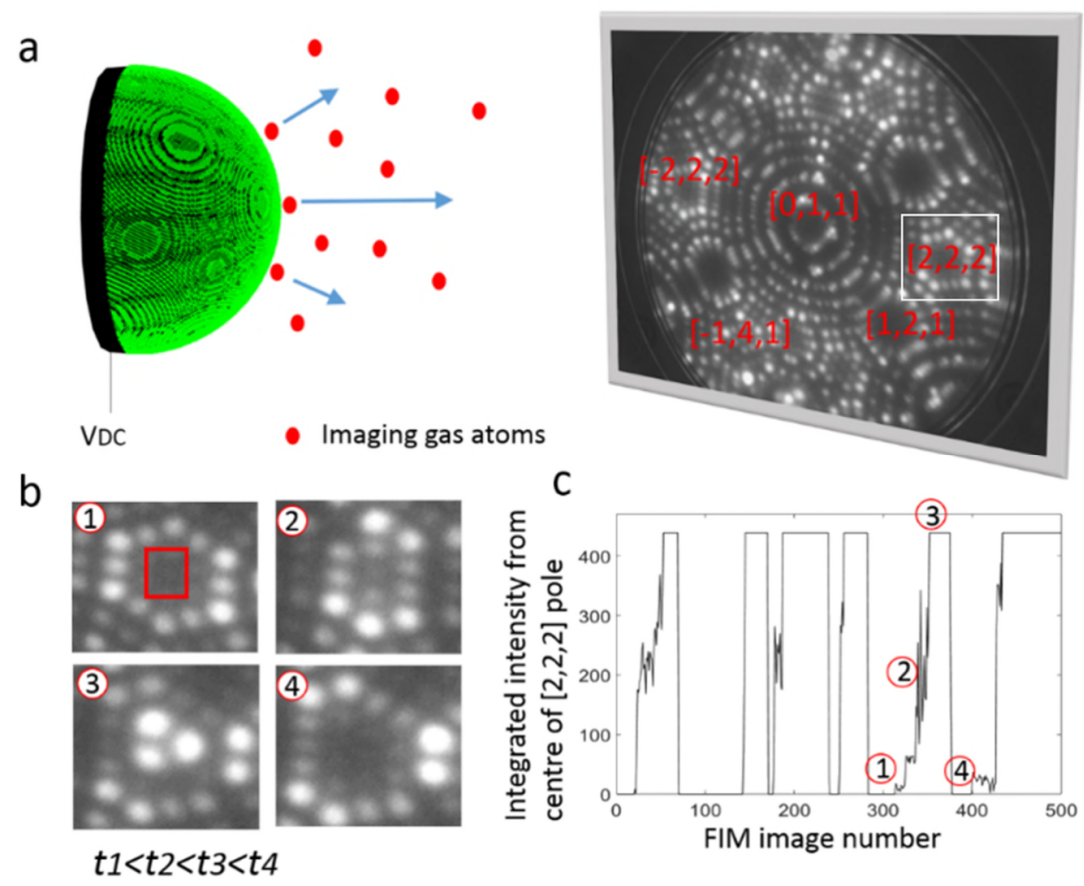

Figure 1 : (a) Schematic illustration of the FIM set-up. Imaging gas atoms are absorbed on the tip of the sample on the left, and are ionized by the electric field towards the phosphor screen on the right. The illustration does not represent the real dimensional scale between the sample and the screen. On the right, FIM image of a tungsten tip with the BCC symmetry evident, and several of the poles indexed. The analysed [2,2,2] pole is marked on the FIM image. (b) Close-up of the [2,2,2] pole. 1-4 are time ordered images describing the evaporation process of a crystallographic plane, beginning from the outside and moving inwards. The square in (1) marks the center of the pole where the integrated intensity in (c) is measured. The plane imaged in (1-3) had been fully evaporated in (4), and the layer below is now exposed. (4) corresponds to a sharp decrease in integrated intensity as can be seen in (c). (c) Integrated intensity from the center of the [2,2,2] pole across 500 FIM images. Each intensity interval of the plot represents the evaporation of one crystallographic layer as a function of image number. Points 1-4 correspond to the contributions from points in time 1-4 marked in (c). 


\section{D reconstruction algorithm}

The reconstruction algorithm consists of the following key steps: Firstly, atomic positions are identified automatically across all images. Next, a tracking algorithm is implemented in order to identify the same atom as it appears in several images across the data set. Finally, the relative crystallographic plane of each atom is determined, completing the coordinates needed to create the final 3D reconstruction. These analysis stages are discussed in details in the following sections.

\section{Gaussian filter and automated detection of atomic coordinates}

In the first stage of the process, a Gaussian filter (GF) is applied to each digital image to remove high frequency noise. The filter used is given by $G F=e^{-\frac{\left(x-\frac{w}{2}\right)^{2}+\left(y-\frac{h}{2}\right)^{2}}{2 \sigma^{2}}}$ where $w, h$ correspond to the width and height dimensions (in pixels) of the image respectively, and $\sigma$ determines the width of GF, which here is set to 40 . The atomically resolved poles of interest are then selected and cropped out to reduce the size of the data and increase calculation speed. In this study, the steps comprising the 3DFIM reconstruction are demonstrated on the $[2,2,2]$ pole, as it evolves on the atomic scale across 7771 FIM images. However, the process can be applied to all poles that exhibit atomic resolution and where the evaporation of atoms can be highly controlled.

Next, within each FIM image the intensity values of all pixels are scanned and the coordinates of the local intensity maxima are identified, as marked by the black dots in figure $2 \mathrm{a}-\mathrm{b}$ (a demonstration of this step on a larger portion of the data can be seen in supplementary video 1 ). These maximum-intensity pixels represent coordinates of all atoms appearing along the stack of images. Note that a threshold value is defined for the intensity peaks to be identified as atoms to avoid false identification due to remaining noise. (In this particular study the threshold was set at approximately 1.5 times the average intensity of background pixels). As can be seen in figure $2 a$, because of this threshold not all atoms on this image have been positively identified. The unidentified atoms are marked manually in figure $2 a$ with white circles for clarity. Local field effects must be taken into consideration here, as these are responsible for atoms being imaged with different intensities. A higher field will result in an atom imaging more brightly, and will also increase the probability of evaporation for that atom. Thus, atoms are usually imaged with the highest intensity directly before evaporation. The evaporation process continues from the image in figure $2 a$ to the point in time represented by figure $2 \mathrm{~b}$. The brightly imaged atoms in figure $2 \mathrm{a}$ have evaporated, as indicated by the red circles in figure $2 a, b$. Subsequent to the evaporation of these atoms, the intensity of the unidentified atoms increases above the identification threshold. Therefore, while not all atoms will be positively identified across all images in which they appear, all atoms will be identified eventually prior to their evaporation.

Figure $2 \mathrm{c}$ plots the coordinates that were automatically detected in the first 80 FIM images, corresponding to the first crystallographic plane to evaporate from the surface of the specimen. To create the plot, the measurement of atomic positions seen in figure $2 a-b$ is repeated across the first 80 images, and the detected atomic coordinates are effectively stacked in the 3D plot. The $z$ coordinate in figure $2 \mathrm{c}$ represents the serial number of the image in which the atoms were identified. For clarity only the first plane is shown here. 

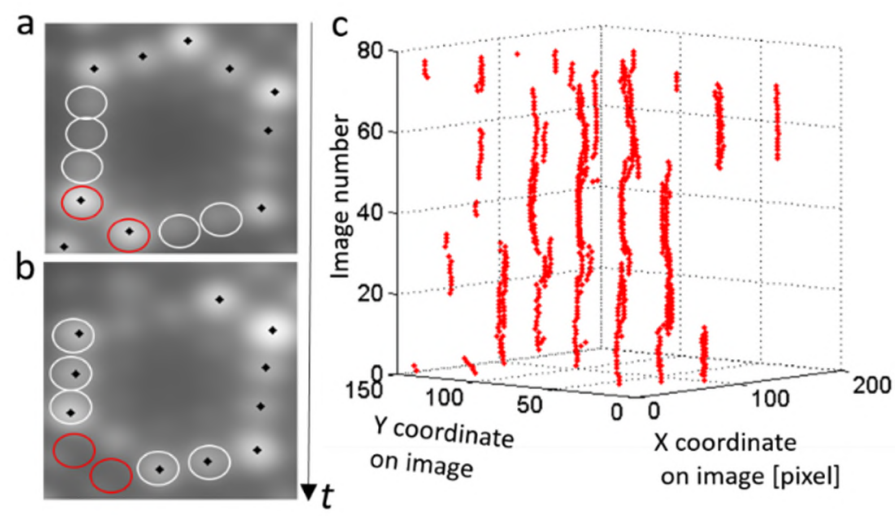

Figure $2:(a, b):$ Time ordered FIM images of the [2,2,2] pole. A Gaussian filter has been applied to remove high frequency noise. Intensity maximum points are identified and marked in black dots. (a) The intensities of atoms marked in white circles are below the set threshold. (b) As the evaporation process progresses, the atoms marked in red circles in (a) have evaporated, and the ones marked in white circles are now imaging with intensities above the identification threshold. (c) All atomic coordinates identified on the first 80 images, corresponding to the evaporation of the first crystallographic layer. The $x-y$ coordinates are set by the pixel coordinates, and the $z$ coordinate at this stage represents the serial number of the image in which every peak was identified.

\section{Consolidating contributions from individual atoms across the sequence}

Once all intensity peaks have been identified across the sequence of images, the next step is to separate these signals into contributions from distinct atoms. The 3D 'point cloud' of information to be analysed (seen in figure 6a) at this stage represents every atom detected across all images, but is not the list of atom coordinates for the final reconstruction. Each atom will typically appear in multiple images from the time that it is first exposed at the surface until it is ultimately evaporated. In fact, the number of images in which each atom appears is dependent on its local field conditions and varies from atom to atom.

The association of a collection of identified intensity peaks to a specific individual atom is carried out through analysis of the relative position of each peak both within the image plane and across the sequence of images. Under the influence of instantaneous field conditions, the imaged positions of atoms may shift from their original recorded positions and their measured position from one image to the next can thus change. The imaging intensity of each atom can also change from image to image, dropping below the threshold for identification at times. These changes (discussed in further detail in the following sections) make it necessary to use an adjustable tracking algorithm to identify and group together intensity points describing the same atom across different images.

First, peaks from all images are separated according to their $x-y$ spatial coordinates (in the image plane). To take into account the displacement of atoms from image to image, a calibration of the imaged size of atoms in pixels is carried out on each image, for each identified intensity peak coordinate. This size is determined here by the edge of a circular area surrounding identified atomic coordinate where peak intensity drops to 0.875 of the respective measured maximum. The defined 'resolution' distance (taken as half the atomic diameter) provides a method for accounting for small displacements in the coordinates of the imaged atoms throughout a series of images. Each atomic coordinate is compared using this calibration distance to all atomic coordinates found on the consecutive image. Intensity peaks that are found to be positioned within the defined $x-y$ 'atom-sized' region will be grouped together at this stage as demonstrated in figure 3a. Each colour represents a group of intensity points associated to the same atom-sized $x-y$ region on the images. Note that atomic positions are compared between adjacent images, 
and not to the initial position in which the atom was first recorded. This is because the image of atoms can gradually move a distance that is comparable to a characteristic atomic radius within the whole course of their exposure to the surface, but is unlikely to move by such a distance between adjacent images.

The distribution of points within each group is then examined with respect to the $z$ direction. Figure $3 b$ shows a close-up of a few such identified groupings. It can be seen that this particular group does not continuously contribute a peak to every image across the sequence. The apparent discontinuities could of course indicate the evaporation of an atom, and the subsequent imaging of another atom from the plane below. Hence, on first inspection the analysis in the $z$ direction would seem to be straight forward: contributions in a sequence of images from the same atom can be separated in the $z$ direction according to the identification of a discontinuity within each $x-y$ group of points. Moreover, it would appear that by combining the integrated intensity measurement, exemplified in figure $1 c$, the relative plane to which each atom originates can be deduced according to the image number in which it was lastly identified before the discontinuity. However, the analysis is not as simple as it would first seem. Frequent deviations from the simple layer-by-layer uniform evaporation sequence result in the need for a more sophisticated analysis and the intervals of integrated intensity in figure $1 \mathrm{c}$ alone cannot provide a reliable determination of the relative plane number for all atoms. Furthermore, instantaneous changes in field conditions, or even residual impurities in the chamber, can also cause the intensity of one atom positively identified in one image to temporarily drop below the detection threshold value in a subsequent image, which would yield a discontinuity such as shown in figure 3b. An example of such an atom 'blinking' between images, is marked with an arrow in the lower close-up in figure $3 b$, but could also extend over a larger interval of images.

The continuously changing imaging intensity therefore necessitates a metric to define the minimum number of images that is likely to separate the imaging of two distinct atoms from different crystallographic planes, as opposed to the blinking of the same atom. Furthermore, this metric should not be a fixed constant across the whole sequence of images, since, as can be seen from figure $1 c$, the evaporation rate at which an entire plane is evaporated varies significantly from plane to plane. To determine the appropriate metric, each intensity peak at the edges of a blinking event is correlated to its position on the integrated intensity plot. The metric required to separate an evaporation event from a blinking event can therefore be assessed with respect to the interval width in the integrated intensity plot. In the example in figure $3 b$, a close-up view reveals that there are two relatively long breaks in the sequence between identification of intensity points in respect to the number of images describing the evaporation of the respective planes. This can be interpreted as two separate evaporation events as occurring in the sequence and the overall imaging of three distinct atoms. Thus, the blue group will be further separated into 3 atoms. In comparison blinking, observable in the close-ups of figure $3 \mathrm{~b}$ is found to be relatively short to the determined metric and therefore the groups of atoms will not be further divided.

Figure $6 \mathrm{~b}$ shows the whole point cloud at the end of the separation procedure. The original intensity points are now coloured by their group number, where each group represents a single atom, imaged across several images. 
a

b
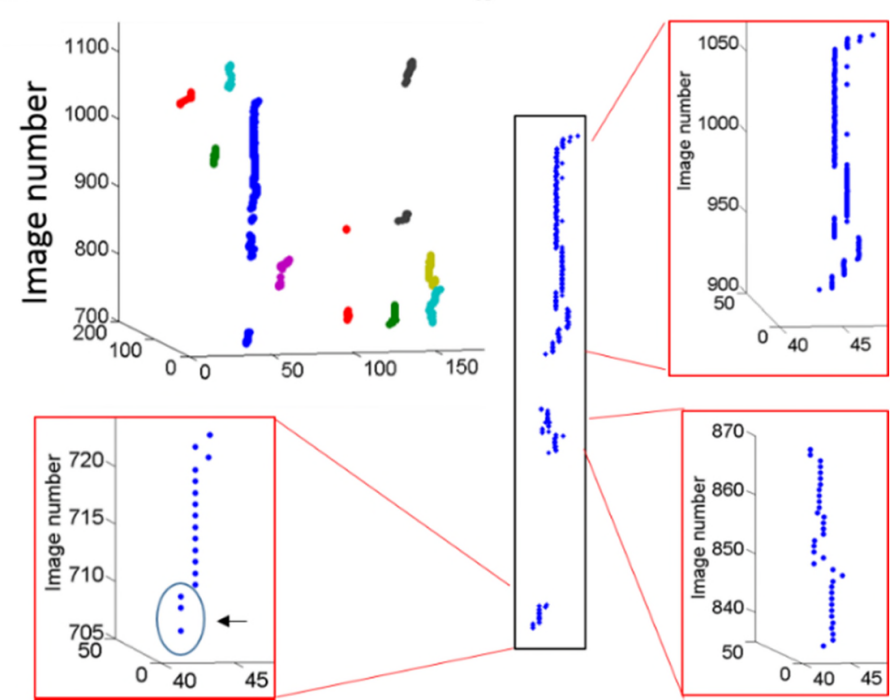

Figure 3 (a) Selected atomic coordinates identified in previous step grouped according to their $x$-y coordinates. Each color represents a group of atomic coordinates that are close enough in their $x$-y coordinates to be suspected as the same atom appearing in different images. (b) Zoom-in view on the blue group of peaks. These are actually three atoms that are placed in the same $x$-y region, in different crystallographic planes. This can be automatically concluded from the long pause in detection across the image series, corresponding to the evaporations of the separate atoms. A short intensity fluctuation 'blinking' event is marked with an arrow.

Finally, once all atoms have been tracked across all images, the total displacement in the recorded coordinates of each atom is calculated (displacement between the last and first recorded positions of the atom) as well as the duration of each atom on the surface (the number of images it appears in). These can then used to identify and resolve 'suspicious' atoms that seem to have very large displacements or very long durations in comparison to the lengths of their correspondent intensity interval in figure $1 \mathrm{c}$.

\section{Separation to crystallographic planes}

The final step in the reconstruction procedure is placing the atoms in their correct crystallographic planes or in other words, correctly determining their in-depth, z, coordinate. Again, this is not a straight forward task due to the frequent divergence from the simplified 'layer-by-layer' evaporation sequence. According to this simplified picture, a kink atom from an upper layer will evaporate prior to an atom in the layer below, and prior to an atom in the centre on the same plane. In this case, all that is needed to determine which atoms belong on the same plane is given by the plot in figure 1c. Each intensity interval on the figure represents the evaporation of one layer, and the atoms can be classified to the different layers according to the number of image in which they were last identified.

However, the true nature of the evaporation sequence is that of a stochastic process. While centre atoms will rarely evaporate prior to a kink atom on the same layer in the absence of a close-by defect [27], it is more common for kink atoms from lower planes to evaporate prior to the removal of the entire atomic layer above them. Examples of this behaviour can be seen in figure 4. In figure 4a, atoms marked with the number ' 2 ' are kink atoms from a lower plane than the three central atoms marked with the number ' 1 '. It is apparent in figure $4 \mathrm{~b}$ that one of the kink atoms, marked with a white arrow, has evaporated prior to the atoms from the plane above. In fact, out of all the atoms that were analysed for this study, 
approximately $27 \%$ were found to be such 'cross-layer' evaporation events. In light of these frequent deviations from an over-simplified 'layer-by-layer' evaporation model, a different approach must be taken to determine the crystallographic plane to assign to each atom.

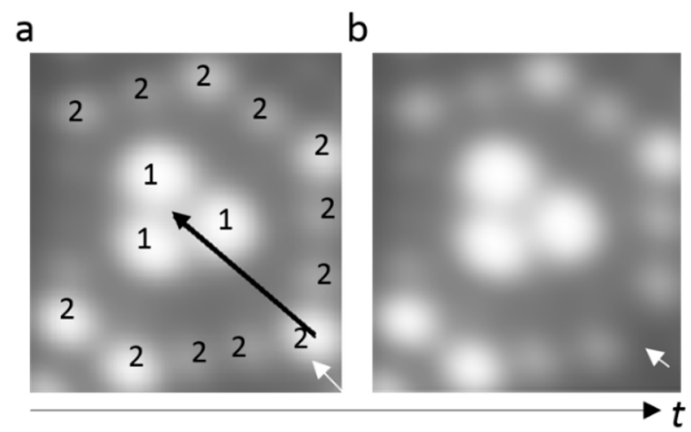

Figure 0 (a) Two planes are imaged here. The final 3 atoms from the higher plane are marked with the number '1', and outer terrace sites from the lower plane are marked with ' 2 '. (b) An atom from a lower layer (marked with a white arrow) is evaporated prior to the three central ones of the upper layer. The two layers can be resolved by detecting decrease in intensity across the black arrow in $a$.

In the example in Figure 0 , the outer terrace of the lower plane can be spatially distinguished from the final 3 centre atoms of the higher plane, by the 'dark' region in the image, separating the two. By examining the intensity profile across the black line in Figure $0 \mathrm{a}$, from the evaporating atom on the outer terrace to the center of the pole, this dark region is evident. Kink atoms evaporated prior to central atoms of higher plane were found to demonstrate highly repetitive profile shapes in several atomic configurations. These have been incorporated into the reconstruction algorithm, and along with derivative behaviours are used to identify such atoms. Figure summarizes intensity profiles of repeating evaporation events. The profiles are always taken across a straight line from the atom in question to the center of the pole. They are calculated according to intensities of pixels along this line, on the image where the highest intensity was recorded for that particular atom (this usually corresponded to the image directly before its evaporation). In Figure a, a characteristic profile is shown for an 'out of sequence' event. This intensity profile clearly demonstrates the decrease in intensity corresponding to the transition across the image from a kink atom belonging to a lower plane to the central atoms contained within the plane above it. For comparison, Figure $b$ shows the same profile for a terrace atom that obeys the simple layerby-layer evaporation pattern, with its final imaging within the correct intensity interval of the plane on which it resides. Unlike the profile in Figure $5 \mathrm{a}$, the decrease in intensity towards the center of the pole is not followed by a subsequent increase, since all the atoms from the plane above have already been evaporated, and thus do not appear in this image. However, the cases presented in Fig $5 \mathrm{c}$ and $\mathrm{d}$, demonstrate an additional complexity to the problem. Atoms in these examples exhibit very similar intensity profiles. However, while the atom the case of Fig $5 \mathrm{c}$ is a central atom evaporating 'in sequence' with its plane, the atom in Fig $5 \mathrm{~d}$ is a kink atom from a lower layer, evaporating 'out of sequence' and ahead of central atoms of the plane above it. In order to distinguish between these cases, it is necessary to examine not only their intensity profiles, but also the corresponding derivatives of these profiles. The derivatives are plotted in the black curves in Figure c,d, along with their respective intensity profiles in red. It can be seen that an additional condition for the identification of an 'out of sequence' kink atom, is 
that the rate of intensity change along the transition between the two planes will be sufficiently large. This condition is specifically illustrated by comparing intensity profiles and their derivatives on the same figure as shown in Figure c-d. The derivative should change significantly enough within the transition region between the two planes so that it then crosses the intensity profile when plotted on the same axes. The algorithm therefore scans for such profiles and derivatives shapes across all atoms examined. Once a 'cross-layer' event is identified, the atom will be assigned to the plane below the one under active consideration.

a

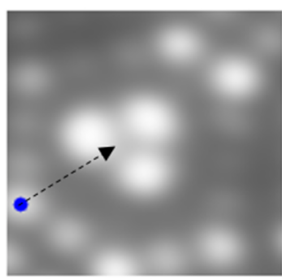

b

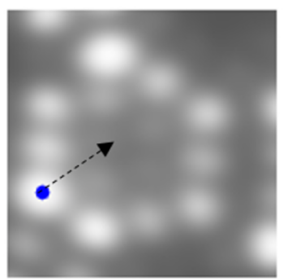

c

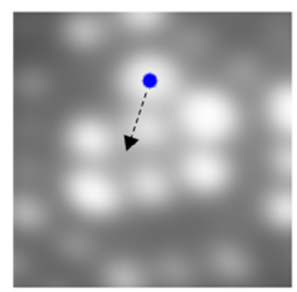

d

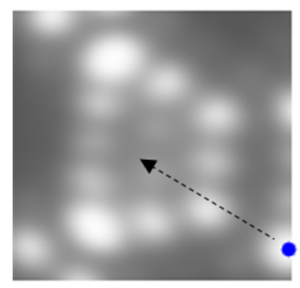

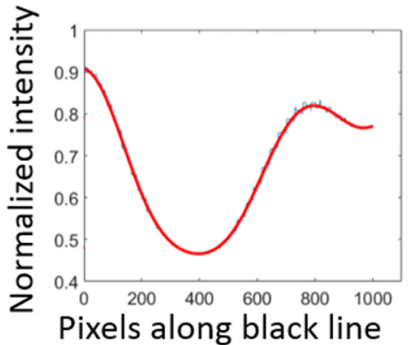
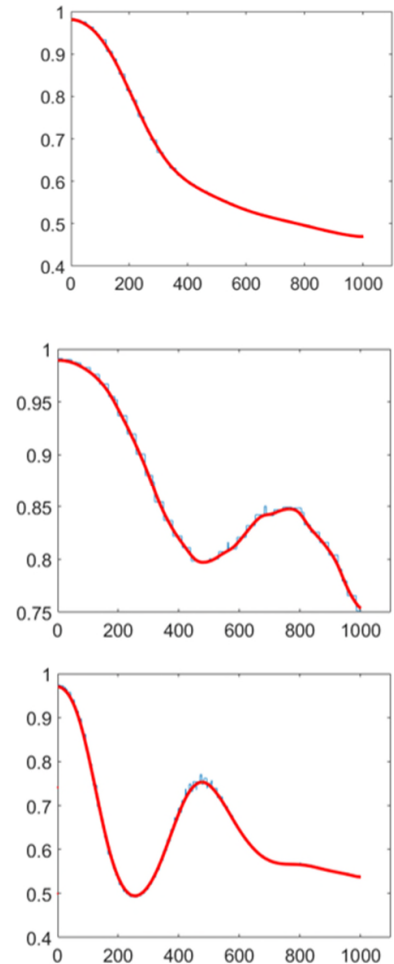

- Intensity

- Derivative
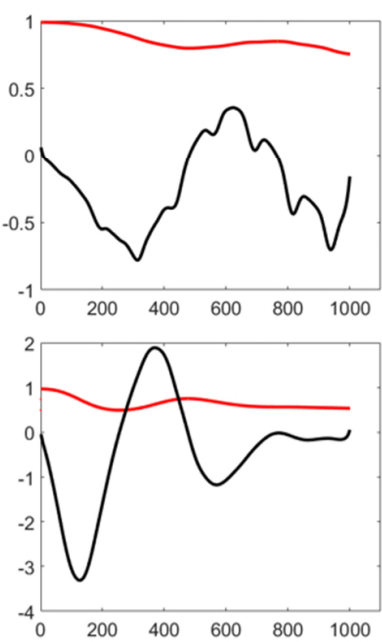

Figure 5 : Characteristic evaporation patterns for kink and central atoms. In red plots are intensity profiles of the atoms marked in blue, across black arrows, connecting the atom to the center of the pole, on their highest intensity image. (a) Terrace atom evaporating 'out of sequence'. (b) Atom evaporating in sequence with it's plane. (c) Central atom evaporating in sequence, exhibiting similar intensity profile to the 'out of sequence' terrace atom in (d). (c) and (d) can be distinguished by the intersection of the intensity and derivative plots along the black arrows (derivatives plotted in black). 
Note that the ' $z$ ' coordinate determined here represents the serial number of the crystallographic layer that the atoms belong to, from the surface and into the depth of the sample, in a direction normal to the plane. Thus, in the conversion from plane numbers to actual $z$ coordinates, each layer will be separated from the next by the theoretical plane constant of the $[2,2,2]$ planes in tungsten. The $x-y$ coordinates can be transformed from 'image dimensions' to real tungsten lattice dimensions by calibrating interatomic distances to the corresponding theoretical values on a tungsten $[2,2,2]$ plane. This procedure is done by finding the average nearest neighbour (NN) distance, measured in terms of number of pixels across the FIM image, between all NNs atoms in the dataset. This distance can then be used to calibrate number of pixels to SI units of length, using the expected theoretical nearest neighbours distance in these planes in tungsten. While the procedure provides an estimation to the corresponding distances, deviations from the resultant calibration are expected due to changes in local magnification conditions, as well as changes to the imaged positions of the atoms prior evaporation (as discussed further in later sections).

Figure 6 summarises all the key stages of this reconstruction approach as applied to all of the analysed images. Figure $6 \mathrm{c}$ presents the final reconstruction, once all atoms have been identified, tracked, and placed in their crystallographic planes. In the final reconstruction all atoms are placed in the initial positions recorded for them, i.e. their coordinates on the image on which they were first identified. The difference between initial and final coordinates can be seen in the 'close-up' view of plane number five, in figure $6 \mathrm{~d}$, where initial positions are marked in blue and final positions in red. Note, that as the final reconstruction is rotated and tilted, additional crystallographic planes other than $[2,2,2]$ are clearly resolvable as demonstrated in figure $6 \mathrm{e}$.
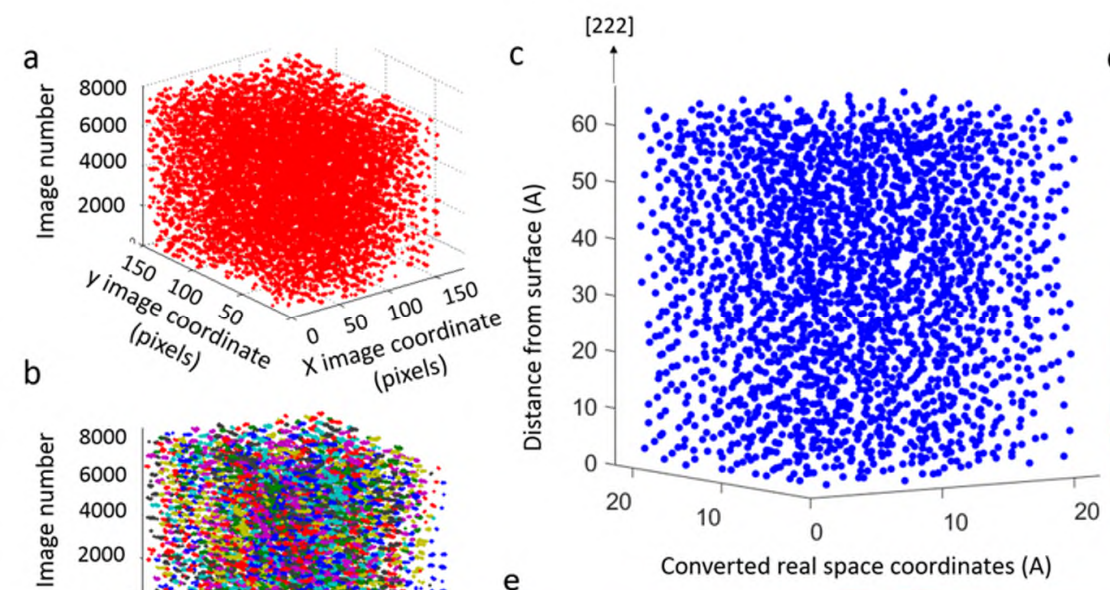

d
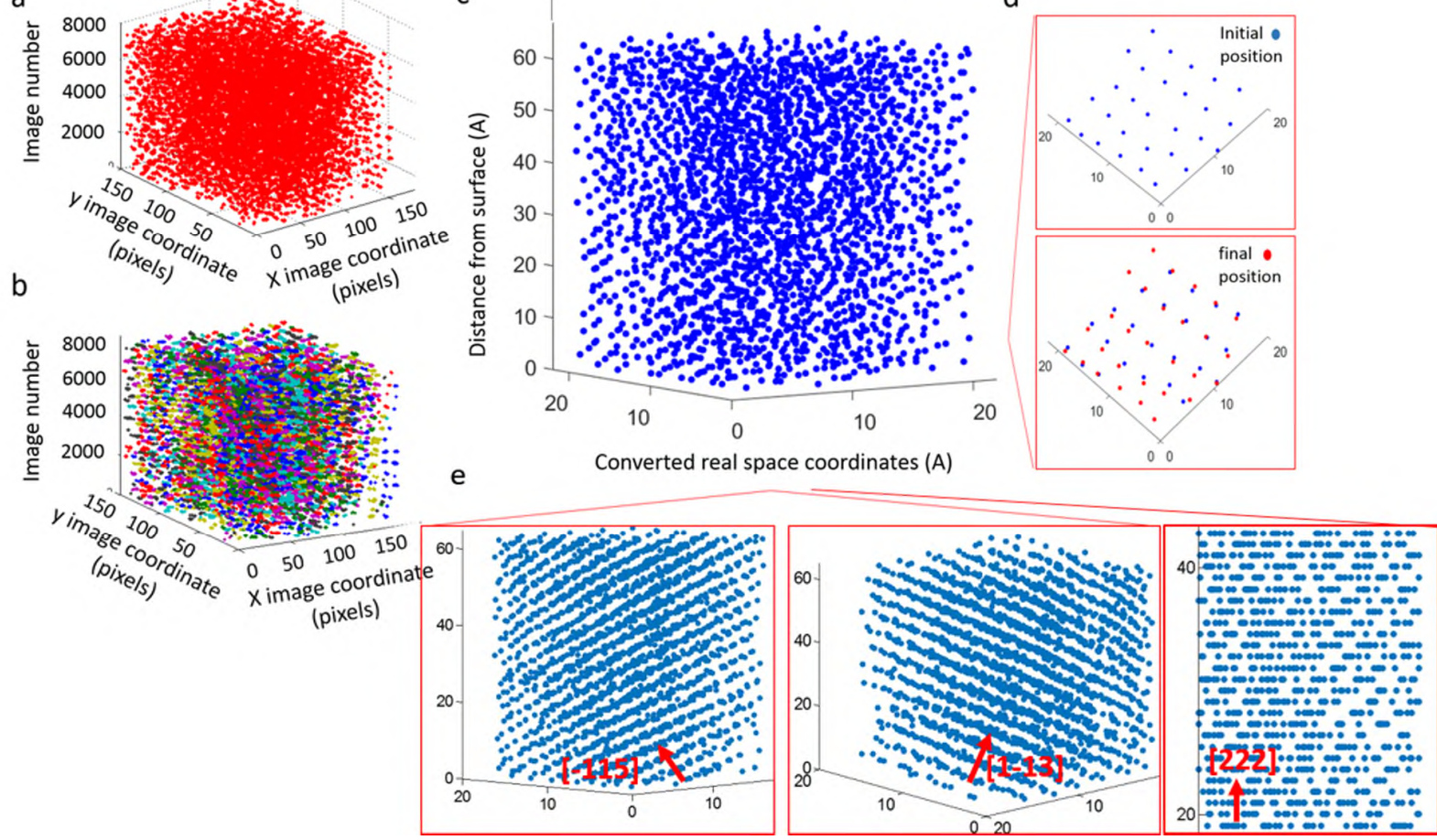

Figure 6: The three steps of reconstruction demonstrated across the entire analysed data set: (a) Intensity peaks are identified on all images. (b) Intensity peaks are grouped according to atoms. (c) Final reconstruction: each atom is positioned in its crystallographic layer in its initial position, coordinates converted to real space. (d) The $5^{\text {th }}$ plane is shown. Initial positions (blue) 
of the atoms on this plane and final positions (in red) are shown together for comparison in the lower window. (e) As the final reconstruction is rotated and tilted, additional crystallographic planes are evident. On the right is a close-up view on some of the reconstructed $[2,2,2]$ planes

\section{Reconstruction of microstructural features}

The reconstruction algorithm described above was designed with the goal of developing a highly accurate approach to the reconstruction of atomically resolved poles, where each atom can be precisely mapped onto the crystal lattice. It is in these volumes where reliable study of vacancies and atomic-sized defects can be performed. Implementation of this algorithm requires high quality data defined by a high level of control in the evaporation process, atomic resolution and a 'layer-by-layer' evaporation. It is thus equally applicable to other poles exhibiting such behavior. Poles that do not exhibit atomic resolution - such as the central $[1,1,0]$ pole of the analysed tungsten sample can be analysed in the same way, however will not provide a final reconstruction that consists of $100 \%$ of the atoms in the analysed volume, since this information is not resolved by the FIM experiment.

The algorithm can also be implemented on FIM datasets that do not possess all of these definitions of high quality data. By applying some straightforward adjustments to the algorithm, larger areas of the field of view can be analysed, as well as a variety of cases exhibiting different imaging contrasts and evaporation characteristics. For example, the approach can be adapted such that a specific microstructure in the data is reconstructed 'atom-by-atom' with this method, as demonstrated in Figure 7 for secondary carbides in a M50 bearing steel. In this case the entire FIM field of view has been incorporated into the analysis, and scanned for atomic coordinates. As the matrix exhibits lower contrast in comparison to the carbides, the threshold for atom identification was chosen so as to include most of the matrix atoms. Since the carbides are imaged much brighter than the selected threshold, the 'blinking' effects described above are less of an issue for the carbide reconstruction, and any 'break' in imaging longer than an experimentally determined number of images is defined to indicate an evaporation event ( 3 images in this case).

As the reconstruction now extends over the entire field of view, the integrated intensity plots cannot be employed across this entire area in a meaningful way. Hence, the depth coordinate for each atom in this case is assigned according to the time at which it is first detected. Alternatively, it could be defined by its evaporation time, similar to the way in which the depth coordinate is assigned in an APT reconstruction. By choosing the relative detection time to define the depth coordinate, retention of these carbides on the surface which is known to bias their reconstruction in APT data [28], is accounted for. Estimation of the tip radius can be used to calibrate $x$ and y pixel coordinates to real space coordinates, and integrated intensity plots measured from around prominent crystallographic poles of the matrix used to approximate the depths evaporated locally. Instead of assuming a constant depth increment between successive images across the whole dataset, the use of intensity plots enables non-uniform evaporation rates to be accounted for in this dataset too. The depth increment between successive images is set as constant only across images from the same intensity interval, and depth calibration is done relative to the evaporation time of the current plane, rather than the whole dataset. Figure $7 \mathrm{~b}$ displays the reconstructed atoms from 500 FIM images, the carbides in red are distinguished from the matrix in yellow). Figure 7c shows the carbides extracted by intensity and density filters, from the reconstruction of 1000 such FIM images, calibrated to real space coordinates. The curvature of the tip and projection effects are not taken into account in the current reconstruction, and should be incorporated into the final stage of the algorithm in 
the future, for cases where large portions of the tip are analysed [29], as opposed to smaller analyses around single crystallographic poles.

a

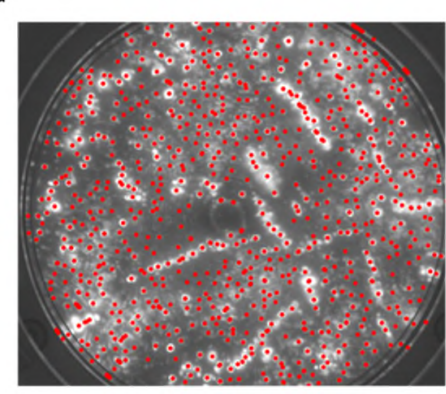

b

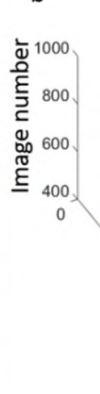

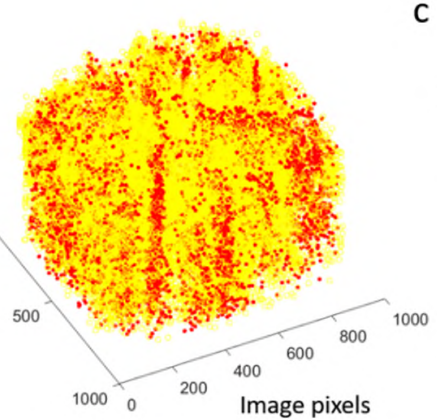

C

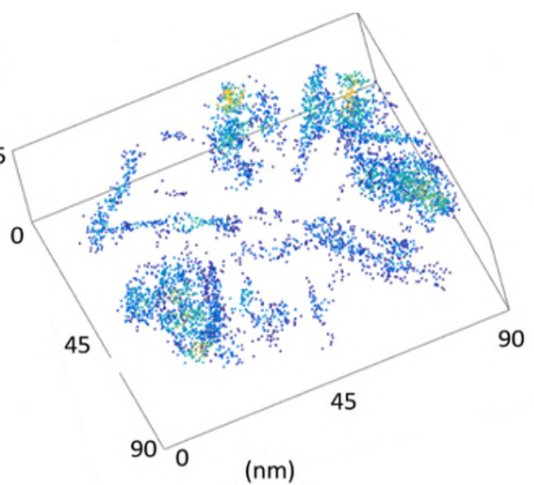

Figure 7: Atom-by-atom reconstruction of carbides in a M50 bearing steel (a) All atoms above a threshold of $40 \%$ maximal intensity are identified. (b) Reconstructed coordinates in image pixels. Red atoms are atoms imaged above an intensity threshold set at 95\% - comprise mostly the carbides in the sample. Yellow atoms are low intensity atoms, corresponding to matrix atoms. (c) High intensity and high density pixels from the reconstruction of 1000 FIM images, calibrated to real space coordinates.

The example in Figure 7 demonstrates the application of the algorithm to a system comprising of a more complex microstructure than the tungsten example, larger reconstructed features, and alloying elements that exhibit different imaging contrast. The resultant 3D reconstruction of the carbides is not as accurate as in the case of the $[2,2,2]$ tungsten pole, as the depth coordinate is assigned by detection time and not a discrete crystallographic plane number. However, the approach still enables an atomistic 3D analysis of the carbides, and the underlying matrix allows direct calibration of the carbides size and relative depth in comparison to atom probe data. Reconstruction of the matrix will yield less accurate results than the carbides in this case, as the threshold for detection of atoms and evaporation events was optimized for the analysis of the carbides. Improved imaging conditions to better capture both carbides and matrix could be achieved by using a mixture of imaging gases [30].

Implementation of the algorithm for the reconstruction of alloys can become challenging as no direct unambiguous chemical information is provided by the FIM experiment. In cases where clear intensity regimes can be identified to separate the different elements in the sample, it is possible to define separate sets of analysis parameters for the optimal reconstruction of all atoms. Automatic detection of out of sequence evaporation events can also be employed to identify different elements. Furthermore, once the data is in the form of a 3D point cloud other post reconstruction analyses can be applied such as nearest neighbour and atomic density measurements. Density measurements can potentially be used to detect precipitates that do not present significant contrast difference to the matrix; discriminate between the carbides in figure 7 for example and adjacent brightly imaged alloying elements in the matrix; and detect depleted features such as those incorporated at grain boundaries. In cases where no differences in intensity, size, or density exist between the elements, or in cases where imaging conditions are significantly different such that atoms are evaporated without being imaged at all (and cannot be resolved by using a mixture of imaging gases), chemical distinction by the algorithm and reconstruction of all atoms will not be possible. 


\section{Measurement of sub-angstrom displacements}

As field conditions change from image to image, lateral displacements in recorded atomic coordinates are observed. These displacements are mentioned above with regards to the reconstruction process as they necessitate a tracking algorithm to identify the same atom across different images. By tracking the recorded positions of atoms across all images in which they appear, it is possible to quantify the series of very subtle displacements of each atom within the images. Measurements of atomic movements in FIM have previously been utilised to great effect to track the successive positions of atoms during surface diffusion before evaporation [31]-[34] . However, the displacements measured in this study represent subtler effects.

A prominent example to such displacements is seen in figure 8 . The last remaining 6 atoms on one of the $[2,2,2]$ planes are shown in a with their peak intensities tracked across a series FIM images (8c), from the point where only those 6 atoms remained on the surface and until the first one of these is evaporated (8b). The position from which this single atom was observed to evaporate is marked by the green circle in figure $8 \mathrm{a}, \mathrm{b}$ while its imaged position is tracked across the series of images and is presented in green in figure 8c. Upon evaporation of this atom, the remaining atoms are imaged in coordinates that are displaced towards its former position, with relative displacement magnitude correlating to their proximity to the evaporated atom. The displacements sizes in figure $8 \mathrm{~b}$ are expressed in units relative to the average nearest neighbour's distance (NN) measured across all images prior to evaporation, with the largest displacements estimated at $0.05 \mathrm{~nm}$ (estimated by calibration to the theoretical nearest neighbour's distance on the $[2,2,2]$ plane). Since the imaged positions of the atoms are constantly changing, even prior to evaporation, the average displacement of the 5 remaining atoms was calculated prior to the evaporation of the green atom across the same range of images. It was found to be $1.8 \%$ of the nearest neighbour's distance, and is considered as the error estimation for the post-evaporation displacements shown in figure $8 \mathrm{~b}$.

The precise origin of these displacements remains un-resolved at this time. One possible explanation is that these are in fact displacements in 'imaged' coordinates originating from artefacts due to how the imaging gas atoms arrange themselves on the surface. Imaging gas atoms adsorbed on atomic sites on the surface will have induced dipole moments, repelling one another, that could cause the adsorbed gas atoms to be slightly displaced outward of the centre of the pole. It is possible that as one of the atoms evaporates, gas atoms adsorbed on the remaining five atoms will relax their positions, and may result in the displacements seen in figure 8 .

An alternative option can be the re-arrangement of the tungsten atoms themselves, after the evaporation of one. Continuous small shifts of atoms are known to be one of the factors limiting the atom probe spatial resolution and contribute to the difficulty of detecting crystallographic features in APT. This phenomena has been anticipated and indirectly quantified in the past [35]. Previous FIM and field desorption studies [36] have indicated atomic movement of the evaporated atom prior to evaporation. Discrepancies between FIM and desorption images of the (110) pole in tungsten demonstrated a roll-up motion that kink ions perform prior to their evaporation, assuming a short-lived metastable position. Similar movements are observed here for atoms neighboring the evaporated atom. If, in fact, due to the movement of tungsten atoms themselves, this type of FIM analysis demonstrates the potential to directly measure and quantify this effect, as well as provide insights into a variety of atomic interactions such as 
the bonding energy of surface atoms, bonding sites, differences between kink and central atoms, and defects effects on all of those.

Finally, the two possible explanations suggested here are not mutually exclusive, and may contribute both to the observed displacements. Possible future experiments to de-couple the two could be done at lower fields and higher temperatures where adsorption effects are reduced.

a

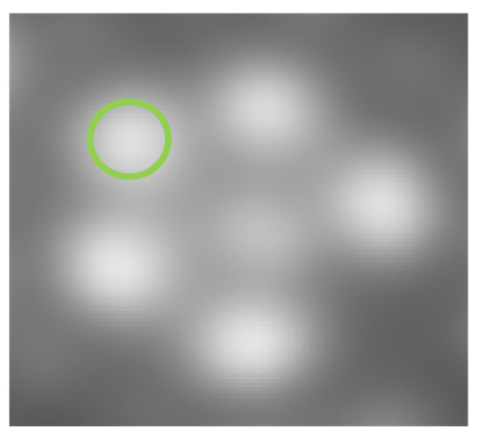

b

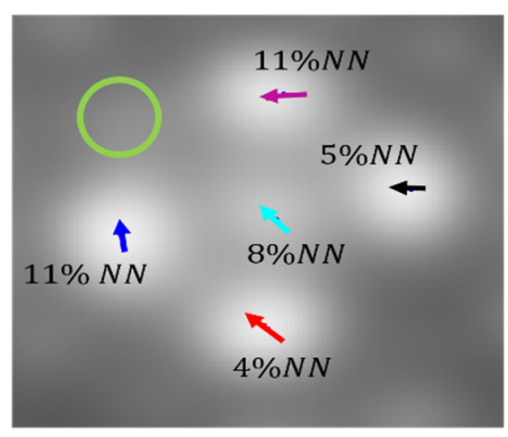

c

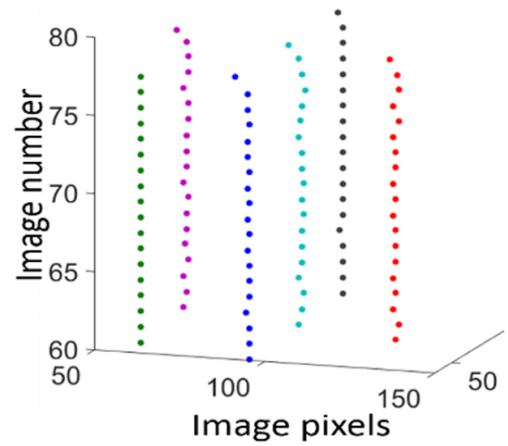

Figure 8: (a) The last six atoms to evaporate from a [2,2,2] layer. The green circle marks the location of the sixth's atom on this plane that is about to evaporate. (b) Remaining atoms and their displacement after the evaporation of the atom marked by the green circle. The arrows mark the displacement direction, all displaced towards the evaporated atom's original location. Colours represent the different atoms, marked with the same colours in $c$. The numbers suggest the extent of the measured displacements relative to the nearest neighbours (NN) distance. (c) The positions of all six atoms as tracked from the moment that they remained only six, and until after the evaporation of the first atom. The first atom to evaporate is marked in green, and corresponds to the position of the green circle in (a). The displacement of the rest of the atoms towards the former position of the green atom can be seen.

\section{Crystal defects}

The reconstruction methodology described in detail in this study has deliberately been developed using a known standard specimen, i.e. a defect-free region in a tungsten lattice. However, this is a significant step towards the automated 3D FIM reconstruction of point defects inside the lattice. An example of such defect can be seen in figure 9 a, where a vacancy is imaged, in a damaged region of our tungsten sample. In this image the presence of a vacancy is visually apparent. However, since instantaneous field distributions determine which atoms will be evident in any given image, it is not possible to confidently identify the presence of a vacancy by observing a single FIM image, but only by analysing the full timeordered sequence. For example, out of sequence evaporation events that can commonly occur, also result in FIM images similar to 9a. These cases can be distinguished from a vacancy only by observing the full time-ordered evaporation sequence of the entire plane.

It is for this reason that the accurate detection and positioning of all plane atoms is essential to the accurate detection of defects, and a simpler 2D analysis of individual images will not suffice. The reconstruction demonstrated in this work is hence an important step towards defects characterization. However, the presence of crystal defects poses additional challenges to the reconstruction algorithm. Figure $9 \mathrm{~b}$ demonstrates the automated reconstruction of a small volume of the lattice where a tri-vacancy cluster is observed. While the reconstruction algorithm as described here is successful in reconstructing 
the damaged volume shown in this particular example, it requires some modifications to robustly reconstruct all damaged regions in an accurate manner. Further work is required on final reconstruction stages where atoms are assigned to a specific plane in depth. In the presence of a vacancy, intensity profiles of the kind in figure 5 utilised by the reconstruction algorithm can have shapes different to the characteristic profiles currently defined to set the atomic layer number. Moreover, if the vacancy happens to be at the centre of the pole and included in the region from which the integrated intensity is calculated, some variation can be expected as well, although this has so far proven to be relatively simple adjustment.
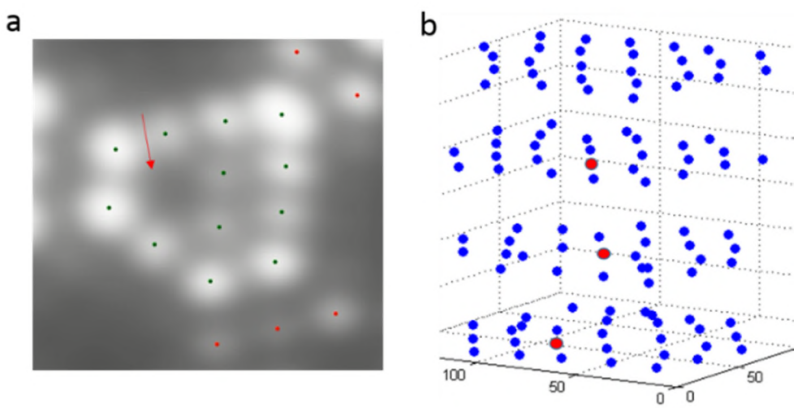

Figure 9: Reconstruction of vacancies: (a) $(-2,2,2)$ layer with a vacancy defect. The algorithm to determine the atomic layers is successful here. Atoms marked in red belong to a lower crystallographic layer than the ones marked in green. (b) Final reconstruction 4 planes containing 3 vacancies. The vacancies are evident in the reconstruction and are marked with a circle.

\section{Conclusion}

In conclusion, the first automated, atomically sensitive reconstruction procedure for 3D field ion microscopy data is described in this work. The technique has the potential to accurately characterize microstructure on the atomic level, detect crystal defects as small as single vacancies and measure displacements in atomic coordinates smaller than $0.05 \mathrm{~nm}$. The digitalization and automation of the analysis enables the characterization of larger volumes of materials in a robust and consistent manner in comparison to the previously employed manual procedure.

The method is demonstrated on a tungsten sample but is in fact based on minimal assumptions and is therefore highly applicable to the analysis of a wide variety of materials, as demonstrated by the reconstruction of carbides in a bearing steel sample.

The stages described in this work set the foundations for the automated reconstruction of 3DFIM data. Future work will be targeted on the reconstruction of damaged microstructures which will rely heavily on these foundations, and will make the necessary adjustments to detect and correctly handle deviations from the un-damaged crystal structure. 
[1] H. Bolt, V. Barabash, G. Federici, J. Linke, a Loarte, J. Roth, and K. Sato, "Plasma facing and high heat flux materials - needs for ITER and beyond," J. Nucl. Mater., vol. 307-311, pp. 43-52, Dec. 2002.

[2] L. K. Mansur, A. F. Rowcliffe, R. K. Nanstad, S. J. Zinkle, W. R. Corwin, and R. E. Stoller, "Materials needs for fusion, Generation IV fission reactors and spallation neutron sources - similarities and differences," J. Nucl. Mater., vol. 329-333, no. 2004, pp. 166-172, Aug. 2004.

[3] Z. Y. Li, N. P. Young, M. Di Vece, S. Palomba, R. E. Palmer, A. L. Bleloch, B. C. Curley, R. L. Johnston, J. Jiang, and J. Yuan, "Three-dimensional atomic-scale structure of size-selected gold nanoclusters," Nature, vol. 451, no. 7174, pp. 46-48, Dec. 2007.

[4] M. Azubel, J. Koivisto, S. Malola, D. Bushnell, G. L. Hura, A. L. Koh, H. Tsunoyama, T. Tsukuda, M. Pettersson, H. Häkkinen, and R. D. Kornberg, "Nanoparticle imaging. Electron microscopy of gold nanoparticles at atomic resolution.," Science, vol. 345, no. 6199, pp. 909-12, Aug. 2014.

[5] R. Xu, C.-C. Chen, L. Wu, M. C. Scott, W. Theis, C. Ophus, M. Bartels, Y. Yang, H. Ramezani-Dakhel, M. R. Sawaya, H. Heinz, L. D. Marks, P. Ercius, and J. Miao, "Three-dimensional coordinates of individual atoms in materials revealed by electron tomography," Nat. Mater., vol. 14, pp. 10991103, Sep. 2015.

[6] E. W. Müller and K. Bahadur, "Field Ionization Of Gases At A Metal Surface And The Resolution Of The Field Ion Microscope," Phys. Rev., vol. 102, no. 3, pp. 624-631, 1956.

[7] S. . Speicher, C.A.; Pimbley, W.T. ; Attardo, M.J; Galligan,J.M; Brenner, "Observation of vacanscies in the field ion microscope," Phys. Lett., vol. 23, no. 3, pp. 3-5, 1966.

[8] D. A. Smith, M. A. Fortes, A. Kelly, and B. Ralph, "Contrast from stacking faults and partial dislocations in field-ion microscope," Philos. Mag., vol. 17, no. 149, pp. 1065-1077, 1968.

[9] M. A. Fortes, D. A. Smith, and B. Ralph, "The interpretation of field-ion micrographs: Contrast from perfect dislocation loops," Philos. Mag., vol. 17, no. 145, pp. 169-176, Jan. 1968.

[10] B. Gault, M. P. Moody, J. M. Cairney, and S. P. Ringer, Atom Probe Microscopy. New York, NY: Springer New York, 2012.

[11] D. N. Seidman, M. I. Current, D. Pramanik, and C. Y. Wei, "Direct observations of the primary state of radiation damage of ion-irradiated tungsten and platinum," Nucl. Instruments Methods, vol. 183, pp. 477-481, 1981.

[12] C. Y. Wei, M. I. Current, and D. N. Seidman, "Direct observation of primary state of damage of ion-irradiated tungsten I. Three-dimensional spatial distribution of vacancies," Philos. Mag. A, pp. 459-491, 1981.

[13] D. N. Seidman, "The study of radiation damage in metals with the field ion and atom probe microscopes," Surf. Sci., vol. 70, pp. 532-565, 1978.

[14] C. Y. Wei and D. N. Seidman, "Direct observation of the vacancy structure of depleted zones in tungsten ion irradiated at 10 K," Appl. Phys. Lett., vol. 34, no. 10, p. 622, 1979.

[15] F. Vurpillot, M. Gilbert, and B. Deconihout, "Towards the three-dimensional field ion microscope," pp. 273-277, 2007. 
[16] F. Danoix, T. Epicier, F. Vurpillot, D. Blavette, C. Tomographic, and A. Probe, "Atomic-scale imaging and analysis of single layer GP zones in a model steel," no. 2012, pp. 1567-1571.

[17] P. Ã. Jessner, R. Danoix, B. Hannoyer, and F. Danoix, "Investigations of the nitrided subsurface layers of an Fe-Cr-model alloy," Ultramicroscopy, vol. 109, no. 2009, pp. 530-534, 2008.

[18] S. Cazottes, F. Vurpillot, A. Fnidiki, D. Lemarchand, M. Baricco, and F. Danoix, "M icroscopy M icroanalysis Nanometer Scale Tomographic Investigation of Fine Scale Precipitates in a CuFeNi Granular System by Three-Dimensional Field Ion Microscopy," pp. 1129-1134, 2012.

[19] F. Vurpillot, B. Gault, B. P. Geiser, and D. J. Larson, "Reconstructing atom probe data: a review.," Ultramicroscopy, vol. 132, pp. 19-30, Sep. 2013.

[20] M. K. Miller, T. F. Kelly, K. Rajan, and S. P. Ringer, "The future of atom probe tomography," Mater. Today, vol. 15, no. 4, pp. 158-165, Apr. 2012.

[21] T. F. Kelly and M. K. Miller, "Atom probe tomography," Rev. Sci. Instrum., vol. 78, no. 3, p. 031101, Mar. 2007.

[22] M. P. Moody, A. V Ceguerra, A. J. Breen, X. Y. Cui, B. Gault, L. T. Stephenson, R. K. W. Marceau, R. C. Powles, and S. P. Ringer, "Atomically resolved tomography to directly inform simulations for structure-property relationships.," Nat. Commun., vol. 5, p. 5501, Jan. 2014.

[23] M. Dagan, L. R. Hanna, A. Xu, S. G. Roberts, G. D. W. Smith, B. Gault, P. D. Edmondson, P. A. J. Bagot, and M. P. Moody, "Ultramicroscopy Imaging of radiation damage using complementary fi eld ion microscopy and atom probe tomography," Ultramicroscopy, pp. 1-8, 2015.

[24] J. W. Davis, V. R. Barabash, A. Makhankov, L. Plöchl, and K. T. Slattery, "Assessment of tungsten for use in the ITER plasma facing components," J. Nucl. Mater., vol. 258-263, no. 1998, pp. 308312, 1998.

[25] R. Neu, R. Dux, A. Kallenbach, T. Pütterich, B. M, J. Fuchs, A. Herrmann, C. Maggi, M. O'Mullane, R. Pugno, I. Radivojevic, V. Rohde, A. Sips, W. Suttrop, A. Whiteford, and the A. U. Team, "Tungsten: an option for divertor and main chamber plasma facing components in future fusion devices," Nucl. Fusion, vol. 45, no. 3, pp. 209-218, 2005.

[26] V. Philipps, "Tungsten as material for plasma-facing components in fusion devices," J. Nucl. Mater., vol. 415, no. 1, pp. S2-S9, Aug. 2011.

[27] K. STILLER and H. O. Andren, "Faulty field evaporation at di-vacancies in $\{222\}$ tungsten," Surf. Sci., vol. 114, pp. 57-61, 1982.

[28] R. K. W. Marceau, P. Choi, and D. Raabe, "Understanding the detection of carbon in austenitic high-Mn steel using atom probe tomography," Ultramicroscopy, vol. 132, pp. 239-247, Sep. 2013.

[29] F. Vurpillot, M. Gilbert, and B. Deconihout, "Towards the three-dimensional field ion microscope," Surf. Interface Anal., vol. 39, no. 2-3, pp. 273-277, Feb. 2007.

[30] J. Akré, F. Danoix, H. Leitner, and P. Auger, "The morphology of secondary-hardening carbides in a martensitic steel at the peak hardness by 3DFIM," Ultramicroscopy, vol. 109, no. 5, pp. 518523, Apr. 2009.

[31] F. Vurpillot, J. Houard, A. Vella, and B. Deconihout, "Thermal response of a field emitter subjected to ultra-fast laser illumination," J. Phys. D. Appl. Phys., vol. 42, no. 12, p. 125502, Jun. 
2009.

[32] G. L. Kellogg, A. F. Wright, and M. S. Daw, "Surface diffusion and adatom-induced substrate relaxations of Pt , Pd, and Ni atoms on Pt ( 001 )," J. Vac. Sci. Technol. A, vol. 9, p. 1757, 1991.

[33] G. L. Kellogg, "Temperature dependence of surface self-diffusion on Pt ( 001 )," Surf. Sci., vol. 246, pp. 31-36, 1991.

[34] S. C. Wang and G. Ehrlich, "Imaging and diffusion of individual iridium adatoms on Ir(111)," Surf. Sci., vol. 224, no. 1989, p. L997, 1989.

[35] B. Gault, M. P. Moody, F. De Geuser, A. La Fontaine, L. T. Stephenson, D. Haley, and S. P. Ringer, “M icroscopy M icroanalysis Spatial Resolution in Atom Probe Tomography," pp. 99-110, 2010.

[36] A. R. Waugh, E. D. Boyes, and M. J. Southon, "INVESTIGATIONS OF FIELD EVAPORATION WITH A FIELD-DESORPTION MICROSCOPE," Surf. Sci., vol. 61, 1976. 